\title{
Relation between extent of coronary artery disease and severity of hyperlipoproteinaemia
}

\author{
R. G. Murray, Ann Tweddel, Jane L. H. C. Third, I. Hutton, W. S. Hillis, \\ A. R. Lorimer, and T. D. V. Lawrie \\ From the Department of Medical Cardiology, Royal Infirmary, Glasgow
}

Lipoprotein analyses were performed in 133 male patients and were correlated with the coronary arteriographic findings. The prevalence of hyperlipoproteinaemia was significantly higher in those patients with coronary artery disease $(P<0.01)$. In addition, the more extensive the degree of coronary artery pathology the higher were the plasma concentrations of total cholesterol, triglyceride, and low density lipoprotein (LDL) cholesterol. Hyperlipoproteinaemia was more prevalent in the younger patients with coronary artery disease $(P<0.02)$.

There is epidemiological evidence to support the association between coronary heart disease and hyperlipoproteinaemia (Kannel et al., 1971; Carlson and Böttiger, 1972). Selective coronary arteriography has provided a means of assessing the degree of coronary atherosclerosis (Kemp et al., 1967). Studies relating arteriographically-proved coronary arterial disease to hyperlipidaemia have been reported (Heinle et al., 1969; Falsetti et al., 1970). Nevertheless, controversy remains over the relation between the extent of the coronary arterial disease and the severity of the plasma lipid abnormality. Some studies show a close relation between cholesterol levels and the severity of the arterial disease (Banks, Raftery, and Oram, 1971 ; Barboriak et al., 1974) while others do not (Cramér, Paulin, and Werkö, 1966; Heinle et al., 1969; Nitter-Hauge and Enge, 1973). Similarly, an association between raised triglyceride concentration and coronary arterial disease has been reported (Salel et al., 1974), but the relation between the extent of vessel disease and triglyceride levels remains in dispute (Cramér et al., 1966; Heinle et al., 1969; Nitter-Hauge and Enge, 1973; Barboriak et al., 1974).

The purpose of this study was to assess the lipoprotein profile in patients having selective coronary arteriography for the diagnosis of chest pain. The lipoprotein abnormalities have been classified and the relation between total cholesterol, triglyceride, and low density lipoprotein cholesterol (LDL) and the extent of vessel disease examined.

Received 30 June 1975.
In addition, the importance of hyperlipoproteinaemia in relation to the age of patients presenting with coronary arterial disease has been assessed.

\section{Patients and methods}

One hundred and thirty-three male patients with chest pain warranting coronary arteriography have been studied. Those with lesions other than coronary arterial disease-for example, aortic valve disease or cardiomyopathy-have been excluded. The mean age of the group was 46 years (range 20 to 63 years).

Selective coronary arteriography was performed by the techniques of Judkins or Sones (Judkins, 1967; Sones and Shirey, 1962). Coronary arterial disease was considered to be present when lumen occlusion was greater than 50 per cent in one or more of the three major vessels (right, left anterior descending, and left circumflex coronary arteries). The patients were accordingly classified as having either a normal vasculature or single, double, or triple vessel disease.

Lipoprotein typing was carried out on two venous blood samples ( $35 \mathrm{ml})$ drawn after a minimum 12-hour, overnight fast. Plasma total cholesterol (Annan and Isherwood, 1969) and triglyceride (Kessler and Lederer, 1966) concentrations were measured. Lipoprotein electrophoresis was carried out using polyacrylamide gel (Narayan, Narayan, and Kummerow, 1965). In 51 patients $(36 \%) 5 \mathrm{ml}$ of plasma was spun for 16 hours at $114000 \mathrm{~g}$. The tubes were sliced and the 'top' $(\mathrm{D}<$ $1.006)$ and the 'bottom' (D>1.006) fractions were obtained. The cholesterol concentrations of the top fraction (very low density lipoprotein (VLDL) and high density lipoprotein (HDL)) were measured. The LDL was precipitated from the bottom fraction by heparin/ 
$\mathrm{MnCl}_{2}$ (Burstein, Scholnick, and Morfin, 1970) and the cholesterol concentration of the remaining HDL was measured. The concentration of LDL cholesterol was obtained by subtracting the HDL cholesterol concentration from the 'bottom' fraction (LDL + HDL) cholesterol concentration.

Using age-related upper limits of normality for cholesterol and triglyceride, as suggested by Fredrickson, Levy, and Lees (1967), the results obtained were classified as follows:

(1) Normal lipoproteins on the basis of normal total cholesterol and triglyceride, a normal electrophoretic pattern, and/or normal LDL, VLDL, and HDL cholesterol concentrations. (2) Type IIa hyperlipoproteinaemia, as defined by a raised total plasma cholesterol, a normal triglyceride, and a pronounced $\beta$ band on electrophoresis or increased LDL cholesterol and normal VLDL and HDL cholesterol concentrations (Beaumont et al., 1970). Type IIb hyperlipoproteinaemia, as defined by an increased triglyceride, usually increased total plasma cholesterol concentration, and intense $\beta$ and pre- $\beta$ bands on electrophoresis or raised LDL and VLDL cholesterol concentration (Beaumont et al., 1970). (3) Type IV hyperlipoproteinaemia, as defined by a raised triglyceride, normal total plasma cholesterol, and a distinct pre- $\beta$ band on electrophoresis or increased VLDL cholesterol concentration (Beaumont et al., 1970).

Types I, III, and V were not encountered in this study.

\section{Results}

Eighteen patients had a normal coronary vasculature on selective coronary arteriography, while 115 patients $(86.5 \%)$ were shown to have significant occlusive lesions affecting one or more vessels. Of those with coronary arterial disease, single vessel disease was found in 27 patients, while almost equal numbers had double and triple vessel disease (Table 1). The distribution of affected vessels is shown in Table 2.

TABLE 1 Coronary arteriogram findings in 133 male patients with chest pain

\begin{tabular}{lr}
\hline Coronary arteriogram & No. \\
\cline { 2 - 3 } Normal vessels & 18 \\
Single vessel disease & 27 \\
Double vessel disease & 42 \\
Triple vessel disease & 46 \\
Total with coronary & 115 \\
\hline
\end{tabular}

\section{Prevalence of hyperlipoproteinaemia}

The distribution of the lipoprotein abnormality is shown in Table 3. Of those with coronary arterial disease 55 per cent had a lipoprotein abnormality whereas only 2 of the 18 patients $(11 \%)$ with normal vessels were affected $(P<0.01)$. Furthermore, hyperlipoproteinaemia was more often encountered in patients with more extensive disease (Fig. 1). The prevalence in patients with double and triple vessel disease $(60 \%)$ was significantly greater than in those with normal or single vessel disease $(27 \%)(\mathbf{P}<0.01)$.

TABLE 2 Distribution of affected vessels in patients with coronary artery disease

\begin{tabular}{llr}
\hline Vessel disease & Vessel affected & No. \\
\hline \multirow{2}{*}{ Single vessel } & LAD & 15 \\
& LCx & 1 \\
& RCA & 11 \\
Double vessel & LAD+RCA & 26 \\
& LAD+LCx & 10 \\
& RCA + LCx & 6 \\
Triple vessel ${ }^{\star}$ & LAD + LCx+RCA 46
\end{tabular}

*Includes two patients with lesions of RCA and left main coronary artery.

$\mathrm{LAD}=$ Left anterior descending coronary artery. LCx $=$ Left circumflex coronary artery. RCA $=$ Right coronary artery.

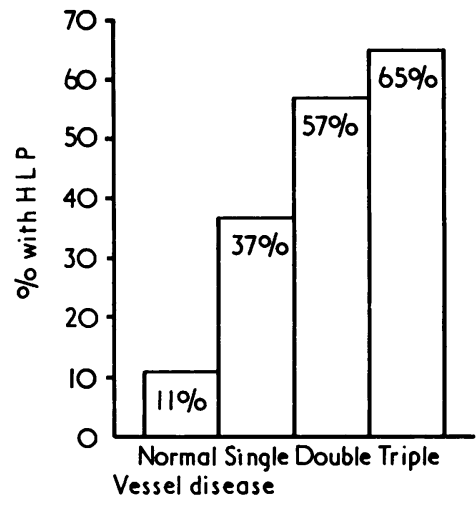

FIG. 1 Correlation of percentage prevalence of hyperlipoproteinaemia (HLP) with results of coronary arteriography in 133 male patients.

\section{Age}

The mean age of the patients with coronary disease was 47 years. To assess the importance of hyperlipoproteinaemia in patients with coronary disease in relation to age, the prevalence in younger patients, defined as less than the mean age of the group studied, was compared with that in older patients (Table 4). Lipoprotein abnormality was encountered 
more often in the younger group of patients $(\mathrm{P}<0.02)$.

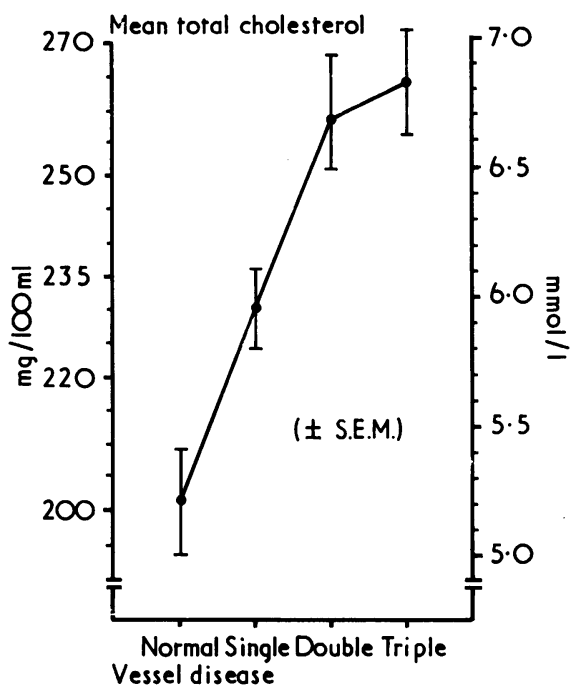

FI G. 2 Relation between mean total plasma cholesterol concentration and results of coronary arteriography in 133 male patients.

Conversion factor from SI to Traditional units: $1 \mathrm{mmol} / \mathrm{l} \approx 38.5 \mathrm{mg} / 100 \mathrm{ml}$.

\section{Cholesterol}

The total mean cholesterol concentration increased with advancing coronary artery disease (Fig. 2). In each group with vessel occlusion (single, double, and triple vessel disease) the total mean cholesterol was significantly higher than in those with normal vessels $(P<0.01$ for all groups). The increment in total mean cholesterol between those with double and triple vessel disease was less, but taken as a group the concentration $6 \cdot 8 \pm 2.3 \mathrm{mmol} / 1 \pm S D$ $(262 \pm 88 \mathrm{mg} / 100 \mathrm{ml})$ was significantly greater than in the combined group of normal and single vessel disease $-5.6 \pm 0.8 \mathrm{mmol} / 1(219 \pm 34.2 \mathrm{mg} / 100 \mathrm{ml})(P$ $<0.01$ ).

In addition, in the younger age group with $\mathrm{CAD}$, as defined above, the total mean cholesterol $6 \cdot 9 \pm 1 \cdot 3$ $\mathrm{mmol} / 1(265 \cdot 6 \pm 49 \cdot 1 \mathrm{mg} / 100 \mathrm{ml})$ was significantly higher than in the older group- $6.3 \pm 1.3 \mathrm{mmol} / 1$ $(242.7 \pm 48.6 \mathrm{mg} / 100 \mathrm{ml})(P<0.01)$.

\section{Triglyceride}

The mean triglyceride concentration also increased with advancing arterial disease (Fig. 3). However, only when two or three vessels were affected was
TABLE 3 Prevalence of hyperlipoproteinaemia (HLP) in 133 male patients having selective coronary arteriography

\begin{tabular}{|c|c|c|}
\hline Coronary arteriogram & No. with $H L P \star$ & Prevalence (\%) \\
\hline $\begin{array}{l}\text { Normal vessels } \\
\text { Coronary artery disease }\end{array}$ & $\begin{array}{c}2(18) \\
63(115)\end{array}$ & $\begin{array}{l}11 \\
55\end{array}$ \\
\hline
\end{tabular}

*Numbers in brackets represent total number of patients in each group.

TABLE 4 Relationship between age and hyperlipoproteinaemia (HLP) in 115 patients with coronary artery disease $(C A D)$

\begin{tabular}{llll}
\hline Age group & No. with CAD & No. with HLP & Prevalence (\%) \\
\hline$<47$ years & 56 & 38 & 68 \\
$>47$ years & 59 & 25 & 42 \\
\hline
\end{tabular}

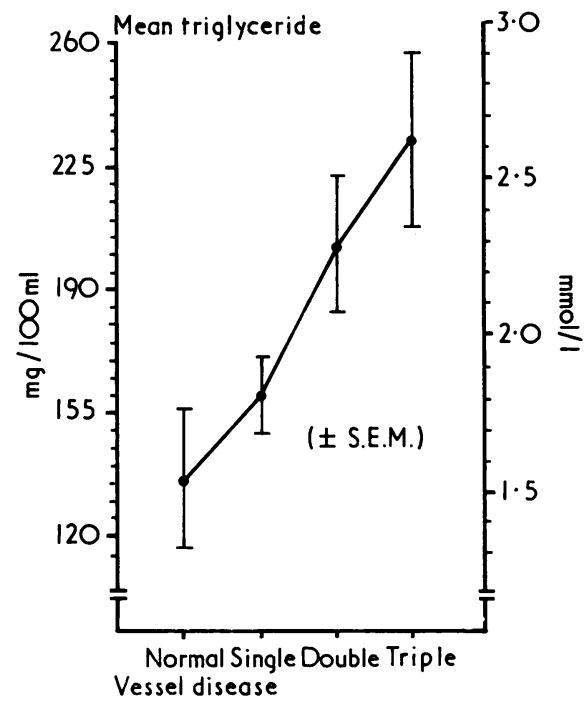

FIG. 3 Relation between mean triglyceride concentrations and results of coronary arteriography in 133 male patients.

Conversion factor from $S I$ to Traditional units: $1 \mathrm{mmol} / 1 \approx 88.5 \mathrm{mg} / 100 \mathrm{ml}$.

the mean triglyceride significantly higher compared with the group with normal vessels $(P<0.025)$. Again comparing those with double and triple vessel, disease and those with normal and single vessel 
disease, the mean triglyceride in the former group$2 \cdot 4 \pm 2 \cdot 2 \mathrm{mmol} / 1(216 \cdot 6 \pm 202 \mathrm{mg} / 100 \mathrm{ml})$-was significantly higher than in the latter group-1.7 \pm 0.0 $\mathrm{mmol} / 1(149.3 \pm 75.2 \mathrm{mg} / 100 \mathrm{ml})(P<0.025)$.

The mean triglyceride in the younger patients$2.6 \pm 1.7 \mathrm{mmol} / 1(227 \pm 154.3 \mathrm{mg} / 100 \mathrm{ml})$-was significantly higher than in the older age group$2.0 \pm 1.3 \mathrm{mmol} / 1(180 \pm 113.2 \mathrm{mg} / 100 \mathrm{ml})(\mathrm{P}<0.05)$.

\section{LDL cholesterol}

Full $\beta$ quantification was performed in 51 patients and the relation between LDL cholesterol and vessel disease examined. There was a significant increase in LDL cholesterol in all three groups with coronary arterial disease compared with those with normal vessels $(P<0.01$ for all groups) (Fig. 4). Also, the mean LDL cholesterol in those with double and triple vessel disease $-4.6 \pm 0.9 \mathrm{mmol} / 1$ $(180.5 \pm 35.2 \mathrm{mg} / 100 \mathrm{ml})$-was higher than in those with normal and single vessel disease-3.8 $\pm 1 \cdot 1$ $\mathrm{mmol} / 1(147.5 \pm 43.7 \mathrm{mg} / 100 \mathrm{ml})(P<0.01)$.

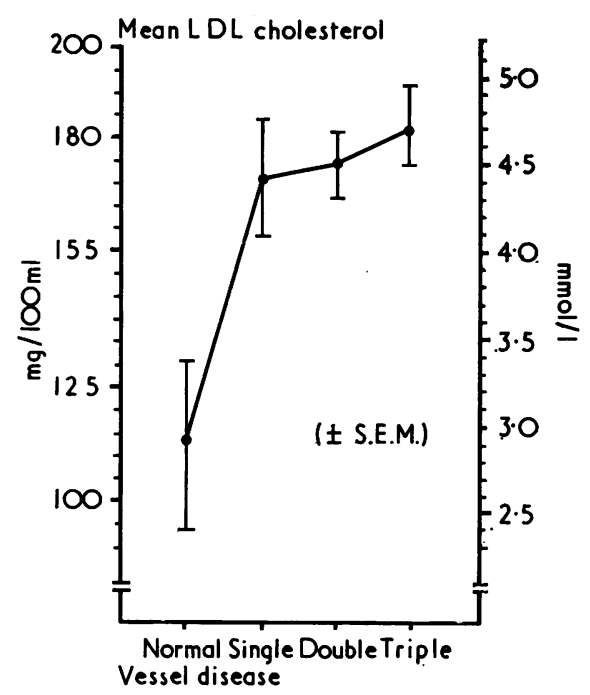

FIG. 4 Relation between mean low density lipoprotein ( $L D L$ ) cholesterol concentration and results of coronary arteriography in 133 male patients.

\section{Lipoprotein typing}

The two patients with normal vessels and hyperlipoproteinaemia both fell into the type IV category (Table 5). Of those with arterial disease type IV was slightly more common than type IIa and $b$, accounting for 54 per cent of lipoprotein abnormalities. However, types IIa and IIb were almost always associated with extensive vessel disease, while 26 per cent of the patients with disease with type IV abnormality had only single vessel involvement. In addition, in those with disease and type II HL.P the combined abnormality of increased LDL and VLDL cholesterol-namely, type IIb-was found in the majority (61\%).

TABLE 5 Correlation between type of hyperlipoproteinaemia (HLP) and results of coronary arteriogram

\begin{tabular}{|c|c|c|c|c|}
\hline \multirow{2}{*}{ Coronary arteriogram } & \multicolumn{3}{|c|}{ HLP type } & \multirow{2}{*}{$\begin{array}{l}\text { Normal } \\
\text { lipoproteins }\end{array}$} \\
\hline & $I I a$ & $I I b$ & $I V$ & \\
\hline $\begin{array}{l}\text { Normal vessels } \\
\text { Single vessel disease } \\
\text { Double vessel disease } \\
\text { Triple vessel disease }\end{array}$ & $\begin{array}{c}- \\
5 \\
6\end{array}$ & $\begin{array}{r}-1 \\
5 \\
12\end{array}$ & $\begin{array}{r}2 \\
9 \\
13 \\
12\end{array}$ & $\begin{array}{l}16 \\
17 \\
19 \\
14\end{array}$ \\
\hline
\end{tabular}

\section{Discussion}

This study shows a significantly higher prevalence of hyperlipoproteinaemia in patients with coronary arterial disease $(55 \%)$ than in those with arteriographically normal vessels. A similar proportion of lipoprotein abnormality in coronary arterial disease has been reported in certain other studies (Heinle et al., 1969-54\%; Barboriak et al., 1974-49\%), while even higher prevalence rates have been recorded (Salel et al., 1974-64\%; Falsetti et al., $1970-71 \%$ ). Such variation may be explained in part by lack of uniformity in the definition of abnormality both in relation to the coronary arteriogram and to lipoprotein abnormalities.

We accept that the group of patients with normal coronary vessels is small and is of a younger mean age than those with disease (39 years of 47 years). Since these are symptomatic patients presenting with chest pain warranting coronary arteriography, they can scarcely be considered truly 'normal'. However, Lorimer et al. (1974) have reported a prevalence of hyperlipoproteinaemia of $12.6 \mathrm{per}$ cent in a group of apparently normal men of a similar age in the West of Scotland compared to 11 per cent found in the normal coronary artery group.

There is a definite relation between total cholesterol, triglyceride, and LDL cholesterol and the extent of vessel disease, the plasma concentration of each increasing with advancing coronary artery 
disease (Fig. 2, 3, and 4). A similar relation between cholesterol and vessel disease has been reported by Banks et al. (1971) and Barboriak et al. (1974). Cramér et al. (1966), however, were unable to show a relation between cholesterol and vessel disease but found raised triglyceride in a significantly higher frequency among patients with severe coronary disease. In contrast, Heinle et al. (1969) and Nitter-Hauge and Enge (1973) were unable to correlate cholesterol and triglyceride concentrations with the extent of vessel disease. LDL cholesterol has seldom been correlated with the coronary arteriographic findings. In contrast to the results in this study, Heinle et al. (1969) reported similar LDL cholesterol levels in patients with and without coronary arterial disease.

Advancing age has been implicated in the development of coronary atherosclerosis. In the younger group of patients with premature vascular disease, however, mean total cholesterol and triglyceride concentrations were significantly higher than in the older age group and, in agreement with certain other authors (Heinle et al., 1969; Barboriak et al., 1974), hyperlipoproteinaemia is more frequent. Clearly this does not detract from the association between age and coronary disease but indicates that hyperlipoproteinaemia carries a high risk of premature coronary disease.

Only three types of hyperlipoproteinaemia were encountered in this study-namely, IIa, IIb, and IV. Type IV hyperlipoproteinaemia was only slightly more common than IIa and $b$ in those with disease but was the most frequent abnormality associated with single vessel disease. The individual numbers in each group are too small to be of statistical value but suggest that type II, and in particular type IIb hyperlipoproteinaemia is more closely linked to extensive vascular pathology.

This study, therefore, confirms the importance of hyperlipoproteinaemia in coronary arterial disease. Mean total cholesterol, triglyceride, and LDL cholesterol concentrations are closely related to the extent of vessel disease. Finally, hyperlipoproteinaemia in the younger patient is an important factor in premature coronary atherosclerosis.

\section{References}

Annan, W., and Isherwood, D. M. (1969). An automated method for the direct determination of total serum cholesterol. Fournal of Medical Laboratory Technology, 26, 202.

Banks, D. C., Raftery, E. B., and Oram, S. (1971). Clinical significance of the coronary arteriogram. British Heart fournal, 33, 863.

Barboriak, J. J., Rimm, A. A., Anderson, A. J., Tristani, F. E., Walker, J. A., and Flemma, R. J. (1974). Coronary artery occlusion and blood lipids. American Heart fournal, 87, 716.

Beaumont, J. L., Carlson, L. A., Cooper, G. R., Fejfar, Z., Fredrickson, D. S., and Strasser, T. (1970). Classification of hyperlipidaemias and hyperlipoproteinaemias. Bullet in of the World Health Organization, 43, 891.

Burstein, M., Scholnick, H. R., and Morfin, R. (1970). Rapid method for the isolation of lipoproteins from human serum by precipitation with polyanions. Fournal of Lipid Research, 11, 583.

Carlson, L. A., and Böttiger, L. E. (1972). Ischaemic heartdisease in relation to fasting values of plasma triglycerides and cholesterol. Lancet, 1,865 .

Cramér, K., Paulin, S., and Werkö, L. (1966). Coronary angiographic findings in correlation with age, body weight, blood pressure, serum lipids and smoking habits. Circulation, 33, 888.

Falsetti, H. L., Schnatz, J. D., Greenc, D. G., and Bunnell, I. L. (1970). Serum lipids and glucose tolerance in angiographically proved coronary artery disease. Chest, $58,111$.

Fredrickson, D. S., Levy, R. I., and Lees, R. S. (1967). Fat transport in lipoproteins-an integrated approach to mechanisms and disorders. New England fournal of Medicine, 276, 34.

Heinle, R. A., Levy, R. I., Fredrickson, D. S., and Gorlin, R. (1969). Lipid and carbohydrate abnormalities in patients with angiographically documented coronary artery disease. American fournal of Cardiology, 24, 178.

Judkins, M. P. (1967). Selective coronary arteriography. Radiology, 89, 815.

Kannel, W. B., Castelli, W. P, Gordon, T., and McNamara, P. M. (1971). Serum cholesterol, lipoproteins and the risk of coronary heart disease. Annals of Internal Medicine, 74, 1 .

Kemp, H. G., Evans, H., Elliott, W. C., and Gorlin, R. (1967). Diagnostic accuracy of selective coronary cinearteriography. Circulation, 36, 526.

Kessler, G., and Lederer, H. (1966). Fluorometric measurement of triglycerides. In Automation in Analytical Chemistry. Technican Symposia 1965, p. 341. Ed by L. T. Skeggs. Medical, New York.

Lorimer, A. R., Cox, F. C., Greaves, D. A., Jubb, J. S., Hawthorne, V. M., Morgan, H. G., and Lawrie, T. D. V. (1974). Prevalence of hyperlipoproteinaemia in apparently healthy men. British Heart fournal, 36, 192.

Narayan, K. A., Narayan, S., and Kummerow, F. A. (1965). Disk electrophoresis of human serum lipoproteins. Nature, 205, 246.

Nitter-Hauge, S., and Enge I. (1973). Relation between blood lipid levels and angiographically evaluated obstructions in coronary arteries. British Heart fournal, 35, 791.

Salel, A. F., Riggs, K., Mason, D. T., Amsterdam, E. A. and Zellis, R. (1974). Importance of type IV hyperlipoproteinaemia as a predisposing factor in coronary artery disease. American fournal of Medicine, 57, 897.

Sones, F. M., Jr., and Shirey, E. K. (1962). Cine coronary arteriography. Modern Concepts of Cardiovascular Disease, 31, 735.

Requests for reprints to Dr. R. G. Murray, Department of Medical Cardiology, The Royal Infirmary, Glasgow G4 0SF. 